\title{
Dinámica Laboral y de Ingresos Durante la Fase de ReCuperación de Argentina (2002-2007)
}

\author{
Fernando Groisman (*)
}

\begin{abstract}
Resumen: En este artículo se analiza la desigualdad, la dinámica laboral y los cambios en la inestabilidad de los ingresos laborales en Argentina entre 2002 y 2007. Dado que la variabilidad de los ingresos de los hogares afecta sus niveles de bienestar se evalúan estos cambios para distintos grupos de hogares. En el documento se analiza también la forma en que las fluctuaciones de los ingresos inciden sobre la desigualda de su distribución. Se utilizan los datos provenientes de la Encuesta Permanente de Hogares (EPH) que lleva adelante el Instituto Nacional de Estadísticas y Censos (INDEC). Los resultados confirman que la movilidad de ingresos tuvo escasa incidencia sobre el nivel de la desigualdad. Simultáneamente la inestabilidad laboral se mostró elevada en aquellos hogares con jefes de bajo nivel educativo. Ello sugiere que la desigualdad en Argentina responde a causas estructurales.
\end{abstract}

Palabras-clave: Inestabilidad Laboral, Inestabilidad de Ingresos, Movilidad, Desigualdad.

\begin{abstract}
This paper analyses inequality, labor market dynamics and changes in the instability of labor incomes in Argentina between 2002 and 2007. Considering that variability of household incomes is important from a welfare perspective the impact of those changes on different household groups is evaluated. The document also analyses how income fluctuations have affected the inequality of income distribution. We use data from the Argentina's Permanent Household Survey (PHS), performed by INDEC. The results obtained confirm that income mobility affected the level of inequality in the income distribution with low intensity. Simultaneously, labor instability revealed high for those households headed by low schooling individuals. This supports the view that inequality in Argentina is due to structural causes.
\end{abstract}

Keywords: Labor Instability, Income Instability, Mobility, Inequality.

\footnotetext{
(*) Investigador del Consejo Nacional de Investigaciones Científicas y Técnicas (CONICET), Argentina. E-mail: fgroisman@tutopia.com. Recebido em: 12.9.08 e aceito em: 28.11.08.
} 


\section{INTRODUCCIÓN}

En este artículo se aborda el análisis de las variaciones de ingresos y la inestabilidad laboral que enfrentaron los hogares entre 2004 y 2007. El propósito perseguido es suministrar evidencias acerca de la magnitud de estos fenómenos en la reciente etapa expansiva y ponderar sus efectos sobre el nivel de la desigualdad. En Argentina las fluctuaciones de ingresos asociadas a la inestabilidad ocupacional han sido rasgos sobresalientes del funcionamiento del mercado de trabajo por lo menos desde fines de la década del ochenta hasta comienzos del corriente decenio. Se ha constatado también que el grado de movilidad - asociado a estas fluctuaciones de ingreso - fue disminuyendo (BECCARIA, GROISMAN, 2006). Una consecuencia directa de ello es que su impacto sobre el nivel de la desigualdad ha sido moderado y decreciente. En el marco de la recuperación económica de los últimos años, que muestra diferencias sustantivas respecto del período previo, es pertinente avanzar en la exploración de esta temática.

Luego de la gran crisis de 2001 Argentina ha mostrado una sostenida recuperación económica que continúa hasta el presente. ${ }^{(1)}$ El desempeño del mercado de trabajo también mostró una evolución acorde. Crecieron el empleo y el poder de compra de los ingresos laborales, se incrementó la proporción de trabajadores registrados y disminuyó la subutilización global de fuerza de trabajo - descendieron la desocupación y la subocupación horaria. En sintonía con este desarrollo se produjo una fuerte reducción de la pobreza absoluta. Sin embargo, la expansión económica generó una moderada disminución en el grado de concentración de los ingresos. La débil respuesta distributiva, relativa al incremento del producto bruto interno, es en sí misma una característica distintiva del período que requiere aún de mayor indagación.

Parte del leve impacto redistributivo obedece ciertamente a un déficit histórico. La heterogeneidad estructural del aparato productivo se encuentra entre las razones del elevado nivel de la concentración de los ingresos. Además, aún cuando en el período 20022007 la elasticidad empleo-producto ha sido elevada, la crónica debilidad de la demanda de empleo frente al abultado excedente laboral tendió a favorecer a los trabajadores con mayor nivel educativo, lo cual conlleva también un efecto desigualador.

Como es sabido, en economías como la argentina, los hogares obtienen mayoritariamente sus ingresos de la actividad laboral que desarrollan sus miembros, por lo tanto, lo que acontezca en este mercado será uno de los principales determinantes de la evolución que siga la concentración de los ingresos. Por lo tanto su indagación no puede quedar marginada de este campo temático y, en este sentido, las consideraciones acerca de la dinámica del empleo al interior de los hogares revisten una evidente importancia. En efecto, las variaciones intertemporales en la condición de ocupación y en los ingresos de los miembros de los hogares tienen efectos directos sobre el bienestar y sobre el nivel de la desigualdad. En el primer caso porque la variabilidad de ingresos acarrea incertidumbre sobre los consumos que pueden enfrentar los hogares. En el segundo caso porque estas fluctuaciones pueden ir acompañadas de alteraciones en la posición relativa que se ocupan en la

(1) Para una descripción del actual régimen macroeconómico en Argentina puede consultarse Frenkel y Rapetti (2008). 
distribución de los ingresos o en la distancia entre ellos. De tal forma que la intensidad de los movimientos - y sus cambios en el tiempo - puede provocar que el nivel de la desigualdad de los ingresos corrientes no refleje adecuadamente el correspondiente a la de los ingresos promedio.

Dada esta relevancia analítica de las variaciones de ingresos para el análisis del nivel y distribución de los mismos, este documento analizará los cambios que ella ha experimentado entre 2004 y 2007, período durante el cual la desigualdad exhibió una marcada estabilidad. Los resultados que aquí se presentan evalúan la intensidad de la inestabilidad laboral y de ingresos según estrato socioeconómico al que pertenecen los hogares. Se evaluó, asimismo, en qué medida los cambios en la variabilidad de los ingresos han tenido un impacto sobre los correspondientes a la distribución del ingreso. ${ }^{(2)}$

Se utilizarán los datos correspondientes al total de los aglomerados urbanos que prove la Encuesta Permanente de Hogares del Indec para los cuatro trimestres de los años 2004, 2005 y 2006 y el primero de 2007 - el último que se ha puesto a disposición de los usuarios.

En la sección siguiente se presentan algunas características de la evolución de la desigualdad y del mercado de trabajo para el período 2002-2007. En la tercera sección se repasan brevemente los diferentes enfoques que se encuentran en la literatura sobre dinámica de los ingresos. En la cuarta se describen la metodología y los datos utilizados. A continuación se presentan los resultados del análisis dinámico. La conclusiones conforman el último apartado.

Algunas características de la concentración de los ingresos y del mercado de trabajo en el período.

\section{LA DESIGUALDAD EN LA DISTRIBUCIÓN DEL INGRESO}

La pobreza ha venido disminuyendo en forma marcada. Sin embargo, esta contracción fue generalizada y no se advirtió más intensa para los hogares con menores recursos — definidos como aquellos con jefe de bajo nivel educativos ${ }^{(3)}$. En realidad, la reducción fue algo mayor para aquellos hogares del estrato superior. Esta performance previene que el resultado redistributivo del crecimiento económico fue de menor cuantía que el que exhibió la reducción de la pobreza.

(2) Son escasos los estudios que han recurrido al análisis dinámico de los ingresos en Argentina. Pueden consultarse Fields y Sánchez Puerta (2005), Albornoz y Menéndez (2002) y Cruces (2003)

(3) Siendo el máximo nivel educativo alcanzado el secundario incompleto. 
Cuadro 1 - Evolución de la pobreza. Total de aglomerados urbanos

\begin{tabular}{|c|c|c|c|c|c|c|c|c|c|c|}
\hline & \multicolumn{2}{|c|}{ Total } & \multicolumn{2}{|c|}{$\begin{array}{l}\text { Hogares con jefe de baja } \\
\text { educación }\end{array}$} & \multicolumn{2}{|c|}{$\begin{array}{l}\text { Hogares con jefe de alta } \\
\text { educación }\end{array}$} & \multicolumn{2}{|c|}{$\begin{array}{c}\text { Hogares con jefe de baja } \\
\text { educación y con edad menor } \\
\text { a } 65 \text { años }\end{array}$} & \multicolumn{2}{|c|}{$\begin{array}{l}\text { Hogares con jefe de alta } \\
\text { educación y con edad menor a } \\
65 \text { años }\end{array}$} \\
\hline & Personas & Hogares & Personas & Hogares & Personas & Hogares & Personas & Hogares & Personas & Hogares \\
\hline II sem -2003 & 48,0 & 36,5 & 60,7 & 24,7 & 48,0 & 18,7 & 65,8 & 25,7 & 57,0 & 20,0 \\
\hline I sem -2004 & 44,4 & 33,5 & 57,7 & 22,1 & 45,6 & 16,5 & 62,5 & 23,0 & 53,5 & 17,8 \\
\hline II sem -2004 & 40,2 & 29,8 & 53,1 & 18,9 & 41,5 & 13,7 & 58,3 & 20,1 & 49,6 & 15,0 \\
\hline I sem -2005 & 38,9 & 28,8 & 51,4 & 18,4 & 39,8 & 13,7 & 56,7 & 19,6 & 47,5 & 15,0 \\
\hline II sem -2005 & 33,8 & 24,7 & 46,5 & 13,9 & 36,0 & 9,9 & 51,8 & 14,7 & 43,3 & 10,7 \\
\hline I sem -2006 & 31,4 & 23,1 & 42,7 & 13,8 & 32,9 & 10,3 & 47,2 & 14,7 & 39,3 & 11,3 \\
\hline II sem -2006 & 26,9 & 19,2 & 38,3 & 10,2 & 28,7 & 7,6 & 42,9 & 10,8 & 34,9 & 8,3 \\
\hline
\end{tabular}

Nota: A partir de 2003 la pobreza se calcula semestralmente. Para este período la última base de datos que el INDEC ha puesto a disposición pública corresponde al segundo semestre del 2006.

FUENTE: Elab. propia sobre datos de EPH-INDEC.

El impacto de la evolución laboral sobre la desigualdad se puede apreciar en forma más directa restringiendo el análisis a los hogares que obtienen básicamente sus ingresos del mercado de trabajo - aquellos con jefes menores de 65 años. La evaluación del coeficiente de Gini confirma una sensible mejora en la equidad al inicio de la fase expansiva de 2002 a 2003 - que luego se moderó. En realidad, la estimación de los intervalos de confianza estadística de este indicador muestra ausencia de diferencias significativas entre las mediciones de 2004 y las posteriores. ${ }^{(4)}$

Cuadro 2 - Evolución de la desigualdad del ingreso per cápita del hogar. Total de aglomerados. Hogares con jefes hasta 65 años

\begin{tabular}{|lrrc|}
\hline & Gini & Limi inf & Lim sup \\
mai/02 & 0,585 & 0,580 & 0,598 \\
II-2003 & 0,554 & 0,545 & 0,570 \\
III-2003 & 0,552 & 0,536 & 0,568 \\
IV-2003 & 0,539 & 0,521 & 0,557 \\
I-2004 & 0,522 & 0,507 & 0,536 \\
II-2004 & 0,518 & 0,505 & 0,531 \\
III-2004 & 0,515 & 0,500 & 0,529 \\
IV-2004 & 0,518 & 0,501 & 0,536 \\
I-2005 & 0,526 & 0,509 & 0,543 \\
II-2005 & 0,516 & 0,503 & 0,529 \\
III-2005 & 0,523 & 0,506 & 0,540 \\
IV-2005 & 0,497 & 0,487 & 0,507 \\
I-2006 & 0,511 & 0,497 & 0,525 \\
II-2006 & 0,488 & 0,476 & 0,500 \\
III-2006 & 0,495 & 0,484 & 0,505 \\
IV-2006 & 0,492 & 0,480 & 0,505 \\
I-2007 & 0,501 & 0,487 & 0,515 \\
FUENTE: Elab. propia sobre datos de EPH-INDEC. \\
\hline \multicolumn{4}{c}{} \\
\hline
\end{tabular}

(4) Obtenida mediante bootsrapping. 


\section{El MERCADO DE TRABaJo}

A partir de 2004, y especialmente desde 2005, la ocupación creció en forma más pronunciada para los miembros de los hogares con jefes de mayor educación. Entre 2002 y 2007 el empleo en los hogares del estrato bajo acumuló un alza del 16\% contra una de $41 \%$ para los integrantes de hogares con jefes más educados. Por su parte, los ingresos laborales mejoraron más para los miembros de hogares de menor educación acumulando un alza entre extremos del $33 \%$ contra el $18 \%$ para los ocupados de hogares comandados por jefes de mayor educación (ver Cuadro 3).

Cuadro 3 - Evolución del empleo y los ingresos. Total de aglomerados. Hogares con jefes hasta 65 años

\begin{tabular}{|c|c|c|c|c|c|c|}
\hline & & Empleo & & & Ingresos re & \\
\hline & Total & $\begin{array}{c}\text { Hasta } \\
\text { secundario } \\
\text { incompleto }\end{array}$ & $\begin{array}{c}\text { Con } \\
\text { secundario } \\
\text { completo }\end{array}$ & Total & $\begin{array}{c}\text { Hasta } \\
\text { secundario } \\
\text { incompleto }\end{array}$ & $\begin{array}{c}\text { Con secundario } \\
\text { completo }\end{array}$ \\
\hline mai/02 & 100,0 & 100,0 & 100,0 & 100,0 & 100,0 & 100,0 \\
\hline II-2003 & 103,8 & 97,7 & 108,1 & 88,0 & 87,8 & 86,8 \\
\hline III-2003 & 108,9 & 105,7 & 112,2 & 94,1 & 91,4 & 93,2 \\
\hline IV-2003 & 110,9 & 106,7 & 115,1 & 97,1 & 97,0 & 94,2 \\
\hline |-2004 & 111,8 & 109,2 & 114,4 & 103,1 & 101,8 & 102,2 \\
\hline II-2004 & 114,3 & 108,4 & 121,1 & 99,3 & 101,0 & 94,6 \\
\hline III-2004 & 116,9 & 110,4 & 124,4 & 98,3 & 98,5 & 94,1 \\
\hline IV-2004 & 118,1 & 112,5 & 124,5 & 101,1 & 102,0 & 96,9 \\
\hline |-2005 & 115,0 & 110,9 & 119,6 & 105,3 & 108,9 & 100,5 \\
\hline II-2005 & 117,5 & 109,6 & 126,7 & 106,5 & 107,0 & 101,9 \\
\hline III-2005 & 121,7 & 111,0 & 134,6 & 114,7 & 109,4 & 110,8 \\
\hline IV-2005 & 122,7 & 116,3 & 130,0 & 111,3 & 112,6 & 106,4 \\
\hline |-2006 & 121,1 & 113,6 & 129,8 & 117,5 & 121,5 & 110,8 \\
\hline II-2006 & 125,7 & 116,3 & 136,7 & 116,2 & 119,8 & 109,1 \\
\hline III-2006 & 126,2 & 114,4 & 140,4 & 119,3 & 123,4 & 110,4 \\
\hline IV-2006 & 127,6 & 115,7 & 141,9 & 121,9 & 124,6 & 113,5 \\
\hline |-2007 & 127,2 & 115,6 & 141,1 & 127,6 & 132,6 & 118,2 \\
\hline
\end{tabular}

Nota: Excluye planes de empleo

FUENTE: Elab. propia sobre datos de EPH-INDEC.

Las menores oportunidades de empleo para los trabajadores de baja calificación se ven refrendadas por otras evidencias. Una de ellas es el elevado nivel de la tasa de desocupación en los hogares con jefe de baja educación. En el primer trimestre de 2007 el nivel de la tasa de desocupación fue de 12\% para los jefes de estos hogares y de $17 \%$ para los miembros no jefes. Por otra parte, si bien mejoró la registración de los jefes de hogar este recupero fue de menor intensidad que el que se produjo en los hogares del estrato más alto. En el mismo año todavía más del 50\% de los miembros de los hogares del estrato bajo no contaba entre sus miembros con un trabajador registrado.

\section{Dimensiones de ANÁliSiS SOBRE LA DINÁMICA DE LOS INGRESOS}

Los estudios sobre las fluctuaciones de ingresos se han concentrado preferencialmente en evaluar el grado en que esas variaciones van acompañadas de cambios en la 
posición que ocupan los perceptores en la distribución del ingreso. ${ }^{(5)}$ Desde esta visión lo relevante es si la ubicación de los perceptores en la distribución del ingreso cambió en el tiempo. En este sentido, uno de los temas que ha predominado en la investigación empírica es la evaluación del impacto de la movilidad de ingresos sobre el nivel de la desigualdad. En efecto, la utilización de un ingreso promedio o de más largo plazo, en lugar de aquellos correspondientes a momentos puntuales, para la medición de la desigualdad conduce a estimaciones diferentes.

Además de la dimensión de análisis que aporta el enfoque de la movilidad otras características de la variabilidad de los ingresos aconsejan incluir la evaluación de la intensidad con que fluctúan los ingresos. Ello responde, fundamentalmente, a que la volatilidad del flujo de recursos corrientes afecta negativamente el bienestar de los individuos al imponer restricciones en su nivel de consumo. Naturalmente, sus efectos son aún mayores cuando las variaciones son imprevistas, y por lo tanto, están acompañadas de incertidumbre.

Como se desprende de lo anterior, ambos enfoques pueden incluso contraponerse. En efecto, podría argumentarse que la dimensión igualadora de los movimientos, y por lo tanto, la desigualdad de más largo plazo podría subestimar las diferencias en los niveles de bienestar que se aproximan con los ingresos estáticos al prescindir de los efectos negativos de la variabilidad de los ingresos. En otras palabras, un dado nivel en la concentración de los ingresos medida en forma estática podría captar mejor la desigualdad del bienestar. Más allá de estas relevantes cuestiones conceptuales existen suficientes argumentos autónomos tanto para la exploración tanto del grado de variación de los ingresos como de las características y efectos de la movilidad sobre la desigualdad.

Otro aspecto a considerar es la propia dinámica de la movilidad de los ingresos, es decir, los cambios en la intensidad de los movimientos de los ingresos. Estos también pueden afectar las evaluaciones que se realicen sobre la evolución de la inequidad. Si no existiesen modificaciones, las mediciones usuales de la desigualdad (con datos de sección cruzada) reflejarán adecuadamente lo que ocurre con la evolución de la concentración de los ingresos.

\section{LOS DATOS Y LOS MÉTODOS UTILIZADOS}

DATOS

Los datos longitudinales - esto es, referidos a los diferentes ingresos que obtiene un mismo hogar a lo largo del tiempo - que se emplearán en este trabajo son los provistos por la Encuesta Permanente de Hogares (EPH) que releva el INDEC. Si bien Argentina no cuenta con encuestas longitudinales, la encuesta mencionada utiliza una muestra de hogares que son reentrevistados en cuatro ocasiones. Ello permite la confección de paneles de observaciones apropiados para abordar el análisis de esas temáticas.

Pueden conocerse los cambios en las variables de interés que cada hogar registra a lo largo de las cuatro ocasiones en que es entrevistado. El relevamiento se realiza en forma

(5) Puede consultarse una síntesis en Ayala y Sastre (2002). 
continua - durante todas las semanas del año - pero las estimaciones se producen trimestralmente. En el esquema de rotación muestral de la encuesta vigente desde 2003 cada hogar es entrevistado en dos ocasiones en dos trimestres consecutivos. Luego es excluido de la muestra durante similar período de tiempo — dos trimestres - y vuelve a ingresar en el relevamiento. En esta segunda etapa cada hogar es visitado nuevamente en otras dos ocasiones también en dos trimestres consecutivos y finalmente abandona la muestra. Ello implica que entre la primera observación y la segunda media un período de tres meses, entre la segunda y la tercera uno de nueve meses y finalmente entre la tercera y cuarta observación un intervalo de tres meses.

Con el propósito de aumentar la cantidad de casos a analizar se procedió a agregar los grupos de rotación en una única base de datos - denominado pooled data. Esto significa que se consideraron simultáneamente a hogares que respondieron a la encuesta en momentos diferentes por lo que se agregaron cambios que se produjeron en períodos cercanos pero distintos. Los datos a emplear corresponden al total de los aglomerados urbanos. En el Cuadro 4 se indica la conformación de los paneles de datos dinámicos a emplear. Se identificaron también dos subgrupos de rotación identificados como Fase 2004-2005 y Fase 2005-2007, respectivamente, para los cuales se realizaron algunas de las estimaciones por separado.

\section{Cuadro 4 - Esquema de datos longitudinales utilizados. Pool de grupos de rotación. Total de aglomerados}

\begin{tabular}{|c|c|c|c|c|c|c|}
\hline & $\begin{array}{l}\text { Primera } \\
\text { observaci }\end{array}$ & $\begin{array}{l}\text { Segunda } \\
\text { observaci }\end{array}$ & $\begin{array}{c}\text { Tercera } \\
\text { observaci }\end{array}$ & $\begin{array}{r}\text { Cuarta } \\
\text { observaci }\end{array}$ & Hogares & Individuos \\
\hline $\begin{array}{l}\text { Fase 2004- } \\
2005\end{array}$ & $\begin{array}{c}\text { Primer trimestre } \\
\text { de } 2004\end{array}$ & $\begin{array}{c}\text { Segundo } \\
\text { trimestre de } 2004\end{array}$ & $\begin{array}{c}\text { Primer trimestre } \\
\text { de } 2005\end{array}$ & $\begin{array}{l}\text { Segundo } \\
\text { trimestre de } 2005\end{array}$ & 2343 & 8200 \\
\hline \multirow[t]{5}{*}{$\begin{array}{l}\text { Fase 2004- } \\
2005\end{array}$} & $\begin{array}{l}\text { Segundo } \\
\text { trimestre de } 2004\end{array}$ & $\begin{array}{c}\text { Tercer trimestre } \\
\text { de } 2004\end{array}$ & $\begin{array}{l}\text { Segundo } \\
\text { trimestre de } 2005\end{array}$ & $\begin{array}{c}\text { Tercer trimestre } \\
\text { de } 2005\end{array}$ & 2449 & 8546 \\
\hline & $\begin{array}{c}\text { Tercer trimestre } \\
\text { de } 2004\end{array}$ & $\begin{array}{c}\text { Cuarto trimestre } \\
\text { de } 2004\end{array}$ & $\begin{array}{c}\text { Tercer trimestre } \\
\text { de } 2005\end{array}$ & $\begin{array}{c}\text { Cuarto trimestre } \\
\text { de } 2005\end{array}$ & 2451 & 8472 \\
\hline & $\begin{array}{c}\text { Cuarto trimestre } \\
\text { de } 2004\end{array}$ & $\begin{array}{c}\text { Primer trimestre } \\
\text { de } 2005\end{array}$ & $\begin{array}{c}\text { Cuarto trimestre } \\
\text { de } 2005\end{array}$ & $\begin{array}{c}\text { Primer trimestre } \\
\text { de } 2006\end{array}$ & 2364 & 8215 \\
\hline & $\begin{array}{c}\text { Primer trimestre } \\
\text { de } 2005\end{array}$ & $\begin{array}{c}\text { Segundo } \\
\text { trimestre de } 2005\end{array}$ & $\begin{array}{c}\text { Primer trimestre } \\
\text { de } 2006\end{array}$ & $\begin{array}{c}\text { Segundo } \\
\text { trimestre de } 2006\end{array}$ & 2366 & 8382 \\
\hline & $\begin{array}{c}\text { Segundo } \\
\text { trimestre de } 2005\end{array}$ & $\begin{array}{c}\text { Tercer trimestre } \\
\text { de } 2005\end{array}$ & $\begin{array}{c}\text { Segundo } \\
\text { trimestre de } 2006\end{array}$ & $\begin{array}{c}\text { Tercer trimestre } \\
\text { de } 2006\end{array}$ & 2453 & 8540 \\
\hline $\begin{array}{l}\text { Fase } 2005- \\
2007\end{array}$ & $\begin{array}{c}\text { Tercer trimestre } \\
\text { de } 2005\end{array}$ & $\begin{array}{c}\text { Cuarto trimestre } \\
\text { de } 2005\end{array}$ & $\begin{array}{c}\text { Tercer trimestre } \\
\text { de } 2006\end{array}$ & $\begin{array}{c}\text { Cuarto trimestre } \\
\text { de } 2006\end{array}$ & 2424 & 8595 \\
\hline $\begin{array}{l}\text { Fase 2005- } \\
2007\end{array}$ & $\begin{array}{c}\text { Cuarto trimestre } \\
\text { de } 2005\end{array}$ & $\begin{array}{c}\text { Primer trimestre } \\
\text { de } 2006\end{array}$ & $\begin{array}{c}\text { Cuarto trimestre } \\
\text { de } 2006\end{array}$ & $\begin{array}{c}\text { Primer trimestre } \\
\text { de } 2007\end{array}$ & 2325 & 8175 \\
\hline
\end{tabular}

El análisis de los ingresos del hogar se restringirá al que obtienen sus miembros por su participación en el mercado de trabajo, sea como asalariados, trabajadores independientes o empleadores. Esta definición acotada de ingresos facilita una más clara asociación de las dinámicas de la desigualdad y de la inestabilidad de ingresos con los factores laborales 
que aparecen como determinantes de las mismas. De esta manera, el ingreso del hogar a utilizar es el que surge de sumar los ingresos laborales de los miembros ocupados.

Al momento de estudiar la inestabilidad de ingresos laborales de los individuos, se analizó lo sucedido con el grupo de aquellas personas ocupadas en al menos una de las observaciones, que son precisamente las que registraron algún ingreso positivo proveniente del trabajo. A efectos de obtener resultados sobre la inestabilidad del poder de compra de los ingresos - el concepto relevante - los montos nominales se corrigieron según las variaciones del Indice de Precios al Consumidor (IPC).

\section{Métodos}

Para la medición de la inestabilidad de los ingresos se recurrió a un indicador clásico como es el coeficiente de variación entre los ingresos que el hogar recibe a lo largo del tiempo. Se midió la variabilidad media que surge de promediar los Coeficientes de Variación $(\mathrm{CV})$ de cada hogar. Se efectuaron las estimaciones de manera desagregada para dos estratos de hogares definidos a partir del nivel de escolaridad del jefe, en tanto esta variable resulta un proxy de la situación socio-económica más permanente.

$$
\begin{aligned}
& C V_{h}=\frac{\sqrt{\sum_{t=1}^{T}\left(y_{h t}-\bar{y}_{h}\right)^{2}}}{\bar{y}_{h}} \\
& y_{h t}=\sum_{i=1}^{m} w_{i h t} \\
& \bar{y}_{h}=\frac{\sum_{t=1}^{T} y_{h t}}{T}
\end{aligned}
$$

donde $\mathrm{T}$ es la cantidad de observaciones que se disponen - en este caso 4 -, "i" representa cada uno de los "m" miembros ocupados del hogar que estuvieron ocupados al menos en uno de las cuatro observaciones.

El coeficiente de variación también se utilize para medir la inestabilidad laboral de los hogares. En este caso se computó la totalidad de ocupados del hogar en cada observación. Para el análisis de la variabilidad ocupacional se recurrió también al análisis de trayectorias laborales según categoría ocupacional.

$\mathrm{El}$ análisis de la intensidad de la movilidad de ingresos, y de los cambios en el tiempo se realizó a partir del estudio de los movimientos que los hogares realizan entre quintiles 
de ingresos entre dos períodos sucesivos. Esta resulta una forma usual de examinar la proporción de perceptores de ingresos que cambian su orden en la distribución.

La dinámica entre cuantiles enfrenta, sin embargo, limitaciones. En particular, no capta los cambios que se producen dentro de los límites de los cuantiles seleccionados. Tampoco resultan adecuados para el análisis de la movilidad absoluta - esto es, no registran las variaciones de ingresos que no acarrean modificaciones de orden. Complementariamente se recurrió a otras medidas de asociación como los coeficientes de correlación simple - Pearson — y de rango — Spearman.

Para cuantificar la influencia de la movilidad sobre la distribución del ingreso se calculó la medida de "ajuste de la desigualdad por movilidad" de Shorrocks (1978) (R) que compara la concentración del ingreso promedio de los T períodos considerados, con el promedio de las desigualdades de esas diversas observaciones.

$$
R\left(W_{T}\right)=\frac{I(\bar{w})}{\sum_{t=1}^{T} \eta_{t} I\left(w_{t}\right)}-1
$$

donde Thace referencia al indicador de desigualdad, w es el ingreso promedio de los $\mathrm{T}$ períodos, $w_{t}$ es el ingreso en el periodo $t$ y $h_{t}$ es un factor de ponderación, definido como la participación de los ingresos totales de las unidades en el período t respecto del ingreso en el conjunto de los T períodos. $R$ tiende a cero como valor máximo cuando no hay movilidad - y va disminuyendo a medida que la movilidad tiene más efectos sobre la distribución.

\section{Resultados Del ANÁLISIS DiNÁMICO}

Se presentan en este apartado algunos resultados que provee el análisis dinámico de los ingresos de los hogares. Se muestra la intensidad de la variabilidad de ingresos y su incidencia entre diferentes grupos de hogares. Se comentan también las características de la inestabilidad ocupacional en el período. Luego se informa el grado de movilidad que estuvo asociado a la dinámica de ingresos comentada y su impacto sobre el nivel de la desigualdad.

\section{LA INESTABILIDAD LABORAL Y DE INGRESOS}

Las fluctuaciones en los ingresos laborales que percibieron los hogares así como las elevadas tasas de entradas y salidas de la ocupación continuaron siendo rasgos característicos del funcionamiento del mercado laboral argentino durante este período. En efecto, los coeficientes de variación — laboral y de ingresos — reflejan elevadas oscilaciones para ambos conceptos (ver Cuadro 5). 
Cuadro 5 - Coeficientes de variación de los ingresos laborales de los hogares y del número de ocupados. Total de aglomerados. Hogares con jefes hasta 65 años

\begin{tabular}{|c|c|c|c|c|c|c|c|c|c|}
\hline \multirow[b]{2}{*}{ Total de hogares } & \multicolumn{3}{|c|}{ Total del pool } & \multicolumn{3}{|c|}{ Fase inicial 2004-2005 } & \multicolumn{3}{|c|}{ Fase final $2005-2007$} \\
\hline & Promedio & Li & Ls & Promedio & $L i$ & Ls & Promedio & $L i$ & Ls \\
\hline Ocupados & 0,21 & 0,21 & 0,22 & 0,21 & 0,20 & 0,23 & 0,21 & 0,20 & 0,22 \\
\hline Ingreso laboral del hogar & 0,34 & 0,34 & 0,35 & 0,34 & 0,32 & 0,35 & 0,34 & 0,33 & 0,36 \\
\hline Hogares con jefes con b & & & & & & & & & \\
\hline Ocupados & 0,24 & 0,23 & 0,24 & 0,23 & 0,21 & 0,25 & 0,24 & 0,22 & 0,25 \\
\hline Ingreso laboral del hogar & 0,37 & 0,36 & 0,38 & 0,36 & 0,34 & 0,38 & 0,38 & 0,36 & 0,39 \\
\hline Hogares con jefes con e & & & & & & & & & \\
\hline Ocupados & 0,18 & 0,16 & 0,19 & 0,18 & 0,16 & 0,21 & 0,16 & 0,14 & 0,18 \\
\hline Ingreso laboral del hogar & 0,29 & 0,28 & 0,30 & 0,30 & 0,28 & 0,33 & 0,28 & 0,26 & 0,30 \\
\hline
\end{tabular}

FUENTE: Elab. propia sobre datos de EPH-INDEC.

Recuérdese que la ausencia de inestabilidad en un hogar — o su inversa, la estabilidad total - implica que el valor del coeficiente de variación sea igual a 0 . El que el promedio de este indicador para la variabilidad ocupacional de los hogares se haya ubicado en 0,21 y el correspondiente a los ingresos laborales en 0,34, en un lapso de tiempo de 15 meses, es evidencia que las fluctuaciones en el volumen de ocupados y en el flujo de los ingresos reales fueron considerables. Otra forma de apreciar ello es si se atiende al hecho de que el $46 \%$ de los hogares mostró cambios en la cantidad de ocupados a lo largo de las cuatro observaciones disponibles.

Más relevante a efectos del análisis distributivo es la comprobación de marcadas diferencias entre los hogares pertenecientes al estrato bajo y alto. Efectivamente, en aquellos con jefe de baja educación la inestabilidad laboral y las fluctuaciones de ingresos fueron, en promedio, alrededor de un tercio más elevadas que en las unidades domésticas cuyo jefe tenía un mayor nivel educativo. La proporción de hogares en los que se produjeron variaciones en la cantidad de ocupados fue del $50 \%$ en el estrato bajo y de $39 \%$ en el estrato alto.

Esta brecha de inestabilidad entre los grupos de hogares analizados no se redujo entre los extremos inicial y final del período reflejando la persistencia de esta desigual exposición al riesgo laboral y de ingresos. Parte de la explicación acerca de esta permanencia parece hallarse en la mejor perspectiva de empleo para aquellos con mayor nivel educativo a lo largo de todo el período que fuera documentada en la sección previa. En otros términos, las menores oportunidades de empleo para los ocupados de bajo nivel educativo habrían conducido también a una mayor incidencia de empleos inestables entre éstos.

Como recurso complementario para evaluar la magnitud de la inestabilidad laboral se analizaron las trayectorias laborales de las personas. Su análisis corrobora el panorama de elevada inestabilidad recién referido. En efecto, en el Cuadro 6 se aprecia que el $38 \%$ de aquellos que estuvieron ocupados en algún momento del período - al menos una vez entre las cuatro ocasiones posibles - exhibió una trayectoria laboral incompleta. En efecto, casi cuatro de cada diez individuos no estuvo ocupado en cada una de las cuatro observaciones realizadas a lo largo de 15 meses. Éste grupo puede subdividirse prácticamente en partes iguales entre aquellos que estuvieron ocupados sólo una vez, en dos ocasiones y en tres oportunidades. 
Cuadro 6 - Características de la inestabilidad laboral. Total de aglomerados. Hogares con jefes hasta 65 años

\begin{tabular}{|c|c|c|c|c|c|c|c|c|}
\hline & $\begin{array}{l}\text { Distribu- } \\
\text { ción }\end{array}$ & $\begin{array}{c}\% \text { de casos } \\
\text { ubicados en el } \\
\text { quintil más pobre } \\
\text { en al menos una } \\
\text { observación }\end{array}$ & $\begin{array}{c}\text { \% de casos } \\
\text { ubicados en los } \\
\text { dos quintiles más } \\
\text { pobres en al } \\
\text { menos una } \\
\text { observación }\end{array}$ & $\begin{array}{l}\text { \% de } \\
\text { baja } \\
\text { educa- } \\
\text { ción }\end{array}$ & $\begin{array}{c}\% \text { de alguna } \\
\text { vez asal no } \\
\text { reg }\end{array}$ & $\begin{array}{l}\% \text { de } \\
\text { varones }\end{array}$ & $\begin{array}{l}\% \text { de } \\
\text { jefes }\end{array}$ & $\begin{array}{l}\text { Promedio de } \\
\text { edad }\end{array}$ \\
\hline Siempre ocupados & 62,0 & 13 & 31 & 48 & 36 & 62 & 62 & 40 \\
\hline Una vez & 2,8 & 47 & 67 & 62 & 54 & 34 & 25 & 36 \\
\hline En la primera observación & 4,1 & 45 & 68 & 65 & 64 & 39 & 12 & 28 \\
\hline En la última observación & 5,6 & 46 & 68 & 62 & 60 & 33 & 17 & 31 \\
\hline Dos veces & 2.9 & 51 & 69 & 61 & 46 & 30 & 33 & 38 \\
\hline En las dos primeras obsen & 4,7 & 42 & 54 & 54 & 64 & 43 & 16 & 30 \\
\hline En las dos últimas & 5,0 & 53 & 74 & 68 & 67 & 46 & 24 & 33 \\
\hline Tres veces & 3,0 & 51 & 71 & 63 & 58 & 40 & 40 & 38 \\
\hline En las tres primeras & 4,2 & 47 & 52 & 58 & 66 & 52 & 31 & 34 \\
\hline En las tres útlimas & 5,6 & 53 & 66 & 66 & 65 & 52 & 41 & 37 \\
\hline Total & 100,0 & & & & & & & \\
\hline
\end{tabular}

FUENTE: Elab. propia sobre datos de EPH-INDEC.

Dado que se trata de una fase económica expansiva es razonable adjudicar la existencia de cierta inestabilidad laboral a la mera creación de empleo. Si bien no es posible aislar este efecto dada la naturaleza de los datos disponibles, se puede realizar una aproximación al mismo. Una forma directa de hacerlo es descontar del grupo de aquellos trabajadores definidos como inestables a los que mostraron transiciones sólo desde la no ocupación a la ocupación, y no en dirección opuesta. El supuesto en este caso es de máxima e implica asumir que quienes se ocuparon no enfrentarán episodios de no ocupación. Luego de este ajuste persisten en la condición de inestabilidad un $60 \%$ de los trabajadores definidos originalmente en esa condición.

La intermitencia laboral estuvo asociada a posiciones desventajosas en la distribución del ingreso lo cual refleja el carácter involuntario que revistió la misma. Se puede apreciar que sólo el 13\% de los que estuvieron siempre ocupados se encontraron en alguna de las cuatro observaciones disponibles en el quintil más pobre de la distribución del ingreso del hogar. Esta incidencia fue superior al $40 \%$ - e incluso al $50 \%$ en gran parte de las trayectorias - para aquellos ocupados inestables. Si se amplía el umbral a los dos quintiles inferiores estos guarismos pasan a ser de un tercio y de dos tercios para los ocupados con trayectorias estables e inestables respectivamente.

Las diferencias también son relevantes en cuanto a la calidad de los puestos de trabajo a los que acceden unos y otros. Entre los que estuvieron siempre ocupados la proporción de aquellos que estuvieron al menos en una ocasión en un puesto precario fue del $36 \%$ mientras que entre los diferentes subgrupos de trabajadores inestables esta incidencia se ubicó en un rango que fue del $46 \%$ al $67 \%$.

La inestabilidad afectó en mayor proporción a aquellos con bajo nivel educativo y fueron más frecuentes entre los no jefes, jóvenes y mujeres. Esta última evidencia es indicativade las limitaciones de los hogares para recurrir al trabajo de otros miembros como mencanismo estabilizador de los ingresos. De todas formas, el hecho de que en promedio un tercio de los trabajadores con trayectorias inestables hayan sido jefes de hogar refleja la extensión de este fenómeno y de sus consecuencias para numerosos hogares.

Otra forma de ponderar el grado en que la baja estabilidad de la condición de ocupación se encuentra asociado a un déficit laboral es a través del análisis de transiciones entre 
diferentes categorías ocupacionales. De su lectura surge un cuadro de situación compatible las características que exhiben los mercados de trabajo segmentados. ${ }^{(6)}$ En el Cuadro 7 se presenta un breve resumen que informa los destinos laborales al cabo de 3, 12 y 15 meses para todos aquellos que estuvieron ocupados en algún momento del período. Allí puede constatarse que quienes no estaban ocupados en la primera observación no lograron, en su mayoría, inserciones laborales estables ni protegidas. En efecto, al cabo de 15 meses el 32\% de éstos volvió a estar no ocupado y el $37 \%$ lo estaba en un puesto precario. Tan sólo el 12\% logró acceder a un puesto asalariado registrado. Similar restricción operó para aquellos que se desempeñaban en un puesto precario al inicio del período. Sólo el 17\% logró el acceso a un puesto protegido 15 meses más tarde, mientras que el 53\% permanecía en un puesto de trabajo no protegido. Entre las posiciones no asalariadas se pudo apreciar una fuerte permanencia en esa condición - 63\%. En este grupo sólo un 5,5\% accedió a una ocupación registrada. En contraste, el $89 \%$ de los asalariados registrados mantuvo esa condición entre puntas del período.

Cuadro 7 - Trayectorias laborales de aquellos alguna vez ocupados. Total de aglomerados. Hogares con jefes hasta 65 años

\begin{tabular}{|c|c|c|c|}
\hline Desde observación inicial & Obs2. (s meses) & ObsS. (12 meses) & Obs4. (15 meses) \\
\hline No asal- no asal & 69,1 & 68,8 & 68,4 \\
\hline No asal - asal reg & 2,5 & 4,7 & 5,5 \\
\hline No asal - asal no reg & 11,6 & 14,0 & $1 s, 1$ \\
\hline No asal - plan & 1,8 & 1,1 & $1, s$ \\
\hline No asal - no ocup & 15,0 & 16,4 & 16,7 \\
\hline Total & 100 & 100 & 100 \\
\hline Asal reg-no asal & $1, s$ & 1,9 & 2,1 \\
\hline Asal reg - asal reg & 92,9 & 89,9 & 89,2 \\
\hline Asal reg - asal no reg & 3,5 & 4,8 & 5,4 \\
\hline Asal reg-plan & 0,0 & 0,0 & 0,0 \\
\hline Asal reg-no ocup & 2,5 & $s, 4$ & $s, s$ \\
\hline Total & 100 & 100 & 100 \\
\hline Asal no reg- no asal & $\mathrm{s}, 2$ & 9,8 & 9,8 \\
\hline Asal no reg-asal reg & 8,1 & 14,9 & 16,9 \\
\hline Asal no reg-asal no reg & $6 S, 2$ & 55,9 & 52,7 \\
\hline Asal no reg - plan & 2,0 & 1,2 & 1,4 \\
\hline Asal no reg-no ocup & 18,5 & 18,1 & 19,5 \\
\hline Total & 100 & 100 & 100 \\
\hline Plan- no asal & 4,1 & 6,1 & 5,7 \\
\hline Plan-asal reg & 0,5 & 1,6 & 2,2 \\
\hline Plan - asal no reg & 9,0 & $1 S, 2$ & 15,9 \\
\hline Plan - plan & 71,0 & 54,2 & 48,5 \\
\hline Plan-no ocup & 15,4 & 24,9 & 28,0 \\
\hline Total & 100 & 100 & 100 \\
\hline No ocup- no asal & 12,0 & 14,5 & 16,5 \\
\hline No ocup - asal reg & 4,4 & 10,8 & 11,8 \\
\hline No ocup - asal no reg & $2 S, 5$ & $S 4,9$ & 57,5 \\
\hline No ocup - plan & 2,6 & 2,8 & 2,8 \\
\hline No ocup - no ocup & 57,6 & $s 7,0$ & $\$ 1,6$ \\
\hline Total & 100 & 100 & 100 \\
\hline
\end{tabular}

FUENTE: Elab. propia sobre datos de EPH-INDEC.

(6) En una línea de análisis emparentada puede verse Tokman (2007). 
Otra forma de apreciar esta dinámica es mediante la identificación de la categoría ocupacional de origen. En el Cuadro 8 se constata que alrededor del $20 \%$ de los asalariados registrados provenía de otra categoría ocupacional, mayoritariamente de posiciones no registradas. También se confirma la elevada proporción de aquellos que efectuaron transiciones entre posiciones no registradas y no ocupación.

Cuadro 8 - Transiciones laborales según categoría de origen.

Total de aglomerados. Hogares con jefes hasta 65 años

\begin{tabular}{|c|c|c|}
\hline Destino & Origen & $\%$ en Obs 4 respecto de Obs 1 \\
\hline \multicolumn{3}{|l|}{ No asal } \\
\hline & De no asal & $11,0 \%$ \\
\hline & De asal reg & $0,7 \%$ \\
\hline & De asal no reg & $2,2 \%$ \\
\hline & De plan & $0,5 \%$ \\
\hline & De no ocup & $5,5 \%$ \\
\hline Subtotal & & $17,7 \%$ \\
\hline & De no asal & $0,9 \%$ \\
\hline & De asal reg & $29,8 \%$ \\
\hline & De asal no reg & $5,8 \%$ \\
\hline & De plan & $0,1 \%$ \\
\hline & De no ocup & $2,5 \%$ \\
\hline Subtotal & & $37,2 \%$ \\
\hline \multicolumn{3}{|l|}{ Asal no reg } \\
\hline & De no asal & $2,2 \%$ \\
\hline & De asal reg & $1,8 \%$ \\
\hline & De asal no reg & $12,2 \%$ \\
\hline & De plan & $0,8 \%$ \\
\hline & De no ocup & $7,9 \%$ \\
\hline Subtotal & & $25,0 \%$ \\
\hline \multicolumn{3}{|l|}{ Plan } \\
\hline & De no asal & $0,2 \%$ \\
\hline & De asal reg & $0,0 \%$ \\
\hline & De asal no reg & $0,5 \%$ \\
\hline & De plan & $2,6 \%$ \\
\hline & De no ocup & $0,6 \%$ \\
\hline Subtotal & & $3,7 \%$ \\
\hline \multicolumn{3}{|l|}{ No ocup } \\
\hline & De no asal & $2,9 \%$ \\
\hline & De asal reg & $1,1 \%$ \\
\hline & De asal no reg & $4,4 \%$ \\
\hline & De plan & $1,5 \%$ \\
\hline & De no ocup & $6,6 \%$ \\
\hline Subtotal & & 16,496 \\
\hline Total & & $100,0 \%$ \\
\hline
\end{tabular}

FUENTE: Elab. propia sobre datos de EPH-INDEC.

En síntesis, aún cuando la mejora de numerosos indicadores laborales ha sido contundente el mercado de trabajo argentino muestra todavía un importante déficit en la demanda de empleo de calidad. En efecto, el panorama recién comentado confirma la existencia de un excedente de oferta laboral que oscila entre la no ocupación y episodios laborales precarios de corta duración. 


\section{Movilidid Y DESIGUALDAD}

Como fue analizado más arriba la desigualdad se redujo en forma moderada entre 2004 y 2007. Al mismo tiempo, se constató la existencia de una elevada variabilidad de ingresos. Usualmente las oscilaciones de los ingresos acarrean cambios en las posiciones relativas de los perceptores y/o en las distancias entre sus ingresos. Esto se verifica, especialmente cuando se tiene en cuenta ciertos eventos laborales, como el paso por el desempleo que implican en muchos casos ausencia de ingresos. El que la desigualdad medida en forma estática no haya exhibido cambios mayores plantea el interrogante acerca de la magnitud de movilidad asociada a esas fluctuaciones.

Para el análisis de la temática de la movilidad de ingresos se recurrió a diversos procedimientos. En primer lugar al análisis de los movimientos que los hogares realizan entre quintiles de ingresos. Ello da una primera aproximación acerca del grado en que las variaciones de ingreso conllevan cambios distributivos. En Cuadro 9 se muestran dos matrices de transición que resumen los cambios entre quintiles de ingreso para un período de 3 meses - entre la observación 1 y 2 - en el primer caso, y para el período más largo de 15 meses - entre la observación 1 y 4 - en el segundo. La comparación de ambas estructuras muestra un panorama de elevada inmovilidad, especialmente elevada en los extremos de la distribución. En efecto, la proporción de aquellos que se mantuvieron en el quintil de ingresos más bajo fue del $71 \%$ en el período corto y del $66 \%$ en el más largo. Para el quintil más rico las valores fueron del $79 \%$ y $76 \%$ para el período corto y largo respectivamente. Si se amplia el rango de posiciones de destino para incluir el quintil inmediato siguiente estos valores se ubican alrededor de $90 \%$. Es decir que los hogares con mayors/menores ingresos al cabo de 15 meses son en una gran proporción los mismos.

Cuadro 9 - Matrices de transición según quintiles del ingreso per cápita de los hogares. Total de aglomerados. Hogares con jefes hasta 65 años

\begin{tabular}{|c|c|c|c|c|c|c|}
\hline \multicolumn{7}{|c|}{ Entre observación 1 y 2 (intervalo de 3 meses) } \\
\hline \multicolumn{7}{|c|}{ Quintil de destino } \\
\hline Quintil inicial & 1 & 2 & 3 & 4 & 5 & Total \\
\hline 1 & 70,6 & 21,5 & 6,8 & 0,8 & 0,3 & 100 \\
\hline 2 & 21,3 & 53,0 & 19,1 & 5,7 & 0,9 & 100 \\
\hline 3 & 6,2 & 20,0 & 51,5 & 19,4 & 3,0 & 100 \\
\hline 4 & 0,7 & 4,0 & 19,2 & 58,6 & 17,5 & 100 \\
\hline 5 & 0,6 & 1,2 & 3,7 & 16,0 & 78,5 & 100 \\
\hline \multicolumn{7}{|c|}{ Entre observación 1 y 4 (intervalo de 15 meses) } \\
\hline \multicolumn{7}{|c|}{ Quintil de destino } \\
\hline Quintil inicial & 1 & 2 & 3 & 4 & 5 & Total \\
\hline 1 & 65,5 & 23,1 & 8,6 & 2,0 & 0,8 & 100 \\
\hline 2 & 25,6 & 44,4 & 21,1 & 7,2 & 1,7 & 100 \\
\hline 3 & 6,3 & 23,8 & 39,7 & 25,7 & 4,4 & 100 \\
\hline 4 & 1,5 & 7,1 & 26,3 & 47,4 & 17,8 & 100 \\
\hline 5 & 0,5 & 1,4 & 4,4 & 18,0 & 75,6 & 100 \\
\hline
\end{tabular}

FUENTE: Elab. propia sobre datos de EPH-INDEC. 
Sin embargo, como ya se mencionó, una limitación de las matrices de transición es que no contabilizan las variaciones de ingresos al interior de los límites de los quintiles. Por lo tanto, complementariamente se analizaron los coeficientes de correlación de Pearson y de Spearman de las distribuciones de los ingresos de los hogares. El Cuadro 10 incluye ambos coeficientes para diferentes intervalos de tiempo. El nivel de la correlación es elevado y es consistente con el panorama de reducida movilidad descripto con anterioridad. No sólo fueron limitados los cambios en el orden de los ingresos - evaluados con el coeficiente de correlación de rango Spearman - sino también en las distancias entre ellos. Además, se aprecia una leve reducción de la asociación a medida que se amplia el período de tiempo que separa a ambas distribuciones. Sin embargo, esta reducción es muy tenue y menos sistemática en la evolución del coeficiente de Pearson. En resumen puede concluirse que el $80 \%$ de los individuos no vieron modificada su posición en en la distribución de los ingresos al cabo de un intervalo de tiempo de 15 meses.

\section{Cuadro 10 - Coeficientes de correlación de los ingresos laborales de los hogares.}

Total de aglomerados. Hogares con jefes hasta 65 años

\begin{tabular}{|lcccc|}
\hline & $\begin{array}{c}\text { Obs. 1 y 2 } \\
\text { (intervalo de 3 } \\
\text { meses) }\end{array}$ & $\begin{array}{c}\text { Obs. 2 y 3 } \\
\text { (intervalo de 9 } \\
\text { meses) }\end{array}$ & $\begin{array}{c}\text { Obs. 1 y 3 } \\
\text { (intervalo de 12 } \\
\text { meses) }\end{array}$ & $\begin{array}{c}\text { Obs. 1 y 4 } \\
\text { (intervalo de 15 } \\
\text { meses) }\end{array}$ \\
\hline Pearson & 0,826 & 0,809 & 0,786 & 0,810 \\
Spearman & 0,852 & 0,828 & 0,813 & 0,801 \\
\hline
\end{tabular}

FUENTE: Elab. propia sobre datos de EPH-INDEC.

La combinación de la evidencia que surge de los dos procedimientos empleados en esta sección puesta en un contexto expansivo de estos años da cuenta de una dinámica laboral con limitados efectos sobre la movilidad de ingresos. Ello resulta consistente con la persistencia de un endurecimiento de las posiciones que ocupan los hogares en la distribución del ingreso. En efecto, una forma de interpretar estos resultados es que los mismos son una de las consecuencias de la fuerte segmentación entre hogares que limita las chances de aquellos con menores recursos para ascender en la escala de ingresos tanto en forma absoluta como relativa. Como fue señalado al analizar las trayectorias laborales se experimentó una elevada inestabilidad que implicó el pasaje por puestos de menor calidad e ingresos. Este comportamiento individual se traslada en buena medida a los hogares dada la escasa capacidad que mostraron para implementar mecanismos de compensación y/o estabilización de los ingresos.

En estos años Argentina ha mostrado una importante variabilidad de los ingresos laborales que se ha manifestado en una elevada inmovilidad — de rango y de distancia. A su vez, se documentó que la desigualdad de la distribución de los ingresos corrientes se mostró estable. Ambas evidencias sugieren que la concentración de los ingresos más permanentes habría seguido una pauta similar y que el efecto de la movilidad de ingresos habría influido sobre el nivel de la desigualdad de la distribución del ingreso en forma marginal. Para cuantificar esta influencia se calculó una medida de "ajuste de la desigualdad 
por movilidad" descripta en sección de metodología. El indicador del grado de desigualdad empleado es, como se señaló más arriba, el coeficiente de Gini.

El ajuste de la desigualdad por movilidad estuvo en torno al 5\% para el conjunto de los hogares. Además, entre los extremos analizados no se hallaron diferencias significativas en la magnitud de este ajuste (ver Cuadro 11). Precisamente, el hecho de que la corrección de la desigualdad estática por movilidad de ingresos haya sido limitada refleja el ya comentado endurecimiento de las posiciones de los hogares en la distribución del ingreso.

\section{Cuadro 11 - Coeficientes Gini de desigualdad de los ingresos de los hogares.Total de aglomerados}

\begin{tabular}{|c|c|c|c|}
\hline & $\begin{array}{c}\text { Total del } \\
\text { pool }\end{array}$ & $\begin{array}{r}\text { Fase inicial } \\
2004-2005 \\
\end{array}$ & $\begin{array}{l}\text { Fase final } \\
2005-2007\end{array}$ \\
\hline \multicolumn{4}{|l|}{ Ingreso per cápita total del hogar (total de hogares) } \\
\hline Gini del ingreso promedio & 0,463 & 0,452 & 0,460 \\
\hline Promedio de Ginis de corte transversal & 0,488 & 0,479 & 0,484 \\
\hline Ajuste por movilidad sobre promedio de Ginis de corte transversal $(\%)$ & $-5,2 \%$ & $-5,8 \%$ & $-4,8 \%$ \\
\hline \multicolumn{4}{|l|}{$\begin{array}{l}\text { Ingreso per cápita laboral del hogar (hogares con jefes menores de } 65 \\
\text { años) }\end{array}$} \\
\hline Gini del ingreso promedio & 0,514 & 0,509 & 0,510 \\
\hline Promedio de Ginis de corte transversal & 0,539 & 0,535 & 0,534 \\
\hline Ajuste por movilidad sobre promedio de Ginis de corte transversal $(\%)$ & $-4,6 \%$ & $-4,9 \%$ & $-4,5 \%$ \\
\hline
\end{tabular}

FUENTE: Elab. propia sobre datos de EPH-INDEC

\section{Conclusiones}

La recuperación económica argentina incluyó una importante recomposición de los niveles de empleo y de los salarios en el marco de una mejora global del funcionamiento del mercado de trabajo. Se incrementó la proporción de trabajadores registrados y descendieron la desocupación y la subocupación horaria. En el plano distributivo se produjo una fuerte reducción de la pobreza absoluta, sin embargo, la desigualdad continuó siendo elevada. Luego de un descenso inicial marcado ese comportamiento se ha visto moderado.

Ante este rumbo distributivo la exploración de lo acontecido en el subperíodo de mayor estabilidad distributiva que va de 2004 a 2007 ha permitido introducir ciertos elementos que parecen tener una incidencia no despreciable sobre este comportamiento. Entre ellos se constata que la dinámica de empleo tendió a favorecer a aquellos con mayor nivel educativo. En efecto, los resultados obtenidos dan cuenta de un panorama en el que los hogares con bajos recursos vieron limitado su acceso a los beneficios de la expansión económica. Los trabajadores de estos hogares dispusieron de menores oportunidades de empleo, y en todo caso, tuvieron acceso puestos de trabajo de menor calidad.

La evidencia con datos dinámicos confirma este diagnóstico. Efectivamente, en aquellos hogares con jefe de baja educación la inestabilidad laboral y las fluctuaciones de ingresos fueron, en promedio, alrededor de un tercio más elevadas que en las unidades domésticas cuyo jefe tenía un mayor nivel educativo. Además, esta brecha de inestabilidad entre los grupos de hogares analizados no se redujo entre los extremos inicial y final del período reflejando la persistencia de esta desigual exposición al riesgo laboral y de ingresos. 
Fernando Groisman - Cadernos PROLAM/USP (Ano 7 - Vol. 2-2008) p. 24-40

La inestabilidad se vio acompañada de una elevada inmovilidad de ingresos. Estos resultados indican que las diferencias de ingresos entre los hogares se mantuvieron a pesar de la mejora económica global. Precisamente, el hecho de que la corrección de la desigualdad estática por movilidad de ingresos haya sido del orden del $5 \%$ refleja este endurecimiento de las posiciones de los hogares en la distribución del ingreso.

Estos elementos parecen sostener el diagnóstico de que la elevada desigualdad en Argentina responde a la persistencia de las dificultades para el acceso a empleos de calidad para los menos calificados. Ello sugiere la necesidad de implementer políticas específicas para su corrección.

\section{REFERENCIAS BIBLIOGRAFICAS}

ALBORNOZ, Facundo; MENÉNDEZ, Marta. Analizing income mobility and inequality: the case of Argentina during the 1990's. Paris, Mimeo, 2002.

AYALA, Luis; SASTRE, Mercedes. La medición de la movilidad de ingresos: enfoques e indicadores. Revista de Economía Pública, Instituto de Estudios Fiscales, v. 162, n. 3, p. 101-131, 2002.

BECCARIA, Luis; GROISMAN Fernando. Inestabilidad, movilidad y distribución del ingreso en Argentina. Revista de la CEPAL, Santiago de Chile, n. 89, LC/G.2312-P/E, 2006.

CRUCES, Guillermo; WODON, Quentin. Risk-adjusted poverty in Argentina:measurement and determinants. World Bank. Argentina Crisis and Poverty. A Poverty Assessment, v. 2, 2003.

FIELDS, Gary; SÁNCHEZ PUERTA, Maria Laura. Earnings mobility in urban Argentina. Background paper prepared for the World Bank, 2005.

FRENKEL, Roberto; RAPETTI, Martín. Five years of competitive and stable real exchange rate in Argentina, 2002-2007. Internacional Review of Applied Economics, n. 22, n. 2, p. 215-226, 2008.

TOKMAN, Victor. Informalidad, inseguridad y cohesión social en América Latina. CEPAL — Serie Políticas sociales, Santiago de Chile, n. 130, 2007. 